\title{
Efecto de la composición sobre el comportamiento frente a la corrosión de aceros inoxidables austeníticos y martensíticos
}

\author{
Natalia Silvina Zadorozne ${ }^{1,2}$, Jorge Darío Vier ${ }^{2}$, Raúl Basilio Rebak ${ }^{3}$ \\ Alicia Esther Ares ${ }^{1,2}$
}

\footnotetext{
${ }^{1}$ IMAM, UNaM, CONICET, FCEQyN, (Calle Félix de Azara No 1552, N3300LQD, Misiones), Posadas, Argentina.

${ }^{2}$ ProMyF - FCEQyN, UNaM, Félix de Azara 1552 (3300), Posadas, Misiones, Argentina

${ }^{3}$ GE Global Research, 1 Research Circle, CEB2551, Schenectady,NY 12309, USA

nataliazadorozne@gmail.com; jorgevier@gmail.com; rebak@ge.com; aares@fceqyn.unam.edu.ar
}

\section{RESUMEN}

El objetivo de este trabajo es estudiar el comportamiento electroquímico de una serie de aceros inoxidables en solución de $\mathrm{NaCl}$ a $10.000 \mathrm{ppm}$ de $\mathrm{Cl}^{-}$y a temperatura ambiente, evaluando las propiedades de acuerdo a las diferencias de estructuras (austenítica y martensítica) y a la composición de las aleaciones, buscando una relación entre su comportamiento y su PREN (Pitting resistance equivalent number). Los aceros inoxidables utilizados fueron: F6NM, 17-4PH, 416 y 304.

Se llevaron a cabo medidas del potencial de circuito abierto en función del tiempo, curvas de polarización potenciodinámica cíclicas, obtención de la resistencia a la polarización por medio de la norma ASTM G59 y medidas de Espectroscopía de Impedancia Electroquímica (EIE).

El análisis de los resultados obtenidos a partir de los ensayos electroquímicos permitió encontrar una relación entre el comportamiento frente a la corrosión de los aceros inoxidables estudiados, su composición y PREN, independientemente de la microestructura que presenta cada uno de ellos.

Palabras clave: Corrosión, Aceros Inoxidables, PREN, picado.

\section{ABSTRACT}

The aim of this work is to study the electrochemical behavior of a series of stainless steels in $\mathrm{NaCl}$ solution $\left(10000 \mathrm{ppm}^{-}\right)$at room temperature.

Considering the differences in structures (austenitic and martensitic) and the composition of alloys, a relationship between their electrochemical behavior and PREN (Pitting resistance equivalent number) was sought. Stainless steels used were: F6NM, 17-4PH, 416 and 304.

Measures of open circuit potential in function of time, cyclic potentiodynamic polarization curves, determining the polarization resistance by ASTM G59 (Tafel) and Electrochemical Impedance Spectroscopy (EIE) were carried out. These studies were complemented by observations of the specimens under optical microscopy.

The analysis of the results, obtained from the electrochemical tests, allowed to find a relationship between the corrosion behavior, its composition and its PREN, independently of the microstructure that having each.

Keywords: Corrosion, Stainless Steel, PREN, pitting.

\section{INTRODUCCIÓN}

La creciente demanda de energía ha llevado a las compañías de exploración y producción de petróleo y gas a extender sus fronteras de explotación e ir a aguas más profundas del mar, lo que implica nuevos desafíos tecnológicos y el uso de materiales que presenten una combinación especial de resistente a la corrosión y resistencia mecánica [1]. Junto con las aleaciones base níquel y el titanio, los aceros inoxidables son una de las familias de aleaciones propuestas como candidatas para satisfacer esta demanda [2,3].

Los aceros inoxidables han sido diseñados para obtener una alta resistencia a la corrosión en un amplio rango de condiciones de servicio. Mientras que su resistencia mecánica está ligada con su microestructura (austeni- 
ta, ferrita, martensita, dúplex y endurecidas por precipitación), su comportamiento frente a la corrosión está asociada a la formación espontánea de una capa de óxido de cromo en la superficie del acero [4-7]. Dentro de las diferentes formas de degradación que se pueden presentar, la corrosión por picaduras es la forma más común en la que se presenta la corrosión localizada en estos materiales $[8,12,13]$. Este tipo de ataque sólo ocurre en presencia de ciertos aniones agresivos. El anión que aparece con más frecuencia es el cloruro. La corrosión localizada por picadura se presenta a partir de un determinado potencial denominado potencial de picado, o en forma más general, potencial de ruptura de la pasividad. El potencial por debajo del cual el desarrollo del ataque localizado se detiene, se denomina potencial de repasivación o de protección. En ciertos sistemas se presenta otro potencial característico, por encima del cual se repasivan las picaduras, denominado potencial de inhibición $[8,12]$.

Se han desarrollado numerosas técnicas electroquímicas y analíticas, junto con novedosos dispositivos experimentales, para el estudio de la corrosión localizada [14,15]. También se han establecido parámetros indicativos del grado de susceptibilidad a la corrosión por picado (y en rendijas) de las aleaciones, tales como PREN ó PRE (Pitting Resistance Equivalent Number), CPT (Critical Pitting Temperature) y CCT (Critical Crevice Temperature) [12, 14, 16-18]. Estos parámetros son funciones de la composición de la aleación. La Ecuación 1 corresponde al parámetro PREN. El mismo fue desarrollado originalmente para aceros inoxidables, y luego modificado para aleaciones base níquel [14, 18, 19]. En la bibliografía se pueden hallar otras expresiones [12].

$$
\mathrm{PREN}=\% \mathrm{Cr}+3,3(\% \mathrm{Mo}+0,5 \% \mathrm{~W})+(16-30) \% \mathrm{~N}
$$

El objetivo de este trabajo es estudiar el comportamiento electroquímico de una serie de aceros inoxi-

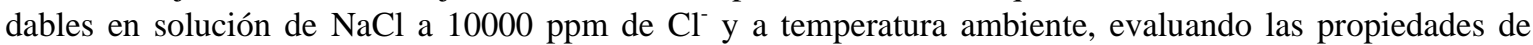
acuerdo a las diferencias de estructuras (austenítica y martensítica) y a la composición de las aleaciones, buscando una relación entre su comportamiento y su PREN (Pitting resistance equivalent number). Los aceros inoxidables utilizados fueron: F6NM, 17-4PH, 416 y 304.

Se llevaron a cabo medidas del potencial de circuito abierto en función del tiempo, curvas de polarización potenciodinámica cíclicas siguiendo la norma ASTM G61[20] para la determinación del potencial de repasivación, obtención de la resistencia a la polarización por medio de la norma ASTM G59 (Tafel) [21] y medidas de espectroscopía de impedancia electroquímica (EIE). Estos estudios se complementaron con observaciones de las probetas mediante microscopia óptica (MO).

El análisis de los resultados obtenidos a partir de los ensayos electroquímicos permitió encontrar una relación entre el comportamiento frente a la corrosión de los aceros inoxidables estudiados y su composición y PREN, independientemente de la microestructura que presenta cada uno de ellos.

\section{MATERIALES Y MÉTODOS}

Se trabajó con probetas de las aleaciones 304, 416 17-4PH y F6NM, cuyas composiciones se presentan en la Tabla 1.

Tabla 1: Composición nominal de las aleaciones.

\begin{tabular}{|c|c|c|c|c|c|c|c|c|c|}
\hline \multirow{2}{*}{ ALEACION } & \multirow{2}{*}{ PREN } & \multirow{2}{*}{ MICROESTRUCTURA } & \multicolumn{7}{|c|}{ COMPOSICION NOMINAL (\% EN PESO) } \\
\hline & & & $\mathrm{Ni}$ & $\mathrm{Cr}$ & Mo & Mn & $\mathrm{Nb}$ & $\mathrm{Cu}$ & $\mathbf{F e}$ \\
\hline 304 & 18 & Austenítico & 8 & 18 & - & - & - & - & Bal. \\
\hline 416 & 13 & Martensítico & - & 13 & - & - & - & - & Bal. \\
\hline $17-4 \mathrm{PH}$ & 17 & Martensítico PH & 4.5 & 16.5 & - & 0.7 & 0.3 & 3.3 & Bal. \\
\hline F6NM & 15 & Martensítico & 4 & 13 & 0.75 & - & - & - & Bal. \\
\hline
\end{tabular}

Se utilizaron probetas en forma de paralelepípedos de dimensiones aproximadas $12 \mathrm{~mm} \times 12 \mathrm{~mm} \mathrm{x}$ $15 \mathrm{~mm}$. Cada una se enroscó a una varilla metálica que se utilizó como contacto eléctrico, la cual se introdujo en un portaprobeta de vidrio y fue aislada de la solución con una junta de PTFE (Politetrafluoretileno). El área de la muestra expuesta a la solución fue de aproximadamente $10 \mathrm{~cm}^{2}$. Todas las probetas fueron pulidas empleando papeles de CSi de granulometría creciente hasta \#1200. Se utilizó una celda electroquímica convencional, un electrodo de referencia de calomel saturado (ECS) $\left(\mathrm{E}_{\mathrm{ECS}}=\mathrm{E}_{\mathrm{ENH}}+0,244 \mathrm{~V}\right)$ y un contraelectro- 
do de platino. El electrodo de referencia se conectó con la solución mediante un capilar de Luggin. En los ensayos se desplazó el oxígeno disuelto en la solución mediante el burbujeo de nitrógeno durante 30 minutos previo a la experiencia, y a lo largo de toda la misma. Los ensayos electroquímicos se realizaron empleando un potenciostato/galvanostato Gamry Reference 600.

Las curvas potenciodinámicas cíclicas se realizaron siguiendo la Norma ASTM G61. Las mismas contaron con tres etapas: 1- Estabilización del sistema a circuito abierto por 1 hora, 2- Barrido anódico desde 100 $\mathrm{mV}$ menos de potencial de corrosión hasta alcanzar una corriente de $1 \mathrm{~mA} / \mathrm{cm}^{2}, 3$ - barrido de potenciales en el sentido catódico hasta alcanzar una corriente negativa. La velocidad de barrido fue de 0,167 mV/s. En los ensayos de EIE, se aplicó una onda de potencial sinusoidal de $5 \mathrm{mV}$ de amplitud eficaz, realizándose un barrido de frecuencias entre $10 \mathrm{kHz}$ y $1 \mathrm{mHz}$, tomando cinco frecuencias por década. Para el ajuste de los resultados se utilizó el método de mínimos cuadrados no lineales diseñado por Bouckamp [22]. Lo anterior se complementó con la observación de las muestras en microscopio óptico.

Las medidas de resistencia a la polarización (Rp) se realizaron por dos métodos: por el Método de Polarización lineal y por Espectroscopía de Impedancia Electroquímica. Para la determinación de las pendientes de Tafel, se obtuvieron las curvas de polarización potenciodinámica en un entorno reducido de potenciales, esto es $50 \mathrm{mV}$ alrededor de potencial de corrosión (Ecorr), tanto en zonas catódicas como anódicas. De acuerdo al Método de Polarización Lineal [23], Rp puede gráficamente obtenerse como la tangente a la curva de polarización en Ecorr, (Ec. (2))

$$
i_{\text {corr }}=\frac{b_{a} \cdot b_{c}}{2,303\left(b_{a}+b_{c}\right)} \frac{1}{R_{p}}
$$

Donde ba y bc son, respectivamente las pendientes de Tafel anódica y catódica.b

Todos los ensayos electroquímicos fueron llevados a cabo en una solución de 10.000 ppm de $\mathrm{NaCl}$ a temperatura ambiente.

\section{RESULTADOS}

La Figura 1 muestra la evolución del potencial de circuito abierto en función del tiempo para los materiales estudiados. Se observó que todas las aleaciones se estabilizaron en aproximadamente una hora, alcanzando los valores finales de - $229 \mathrm{mV}_{\mathrm{ECS}}$ la aleación F6NM, -236 mV $\mathrm{ECS}_{\mathrm{E}}$ la aleación 17-4PH, - $292 \mathrm{mV}_{\mathrm{ECS}}$ la aleación 304 y -362 mV $\mathrm{ECS}_{\mathrm{ECS}}$ la aleación 416. Esta última mostró una variación errática del potencial, mientras que las demás materiales tuvieron una evolución creciente y hasta estabilizarte en los valores correspondientes.

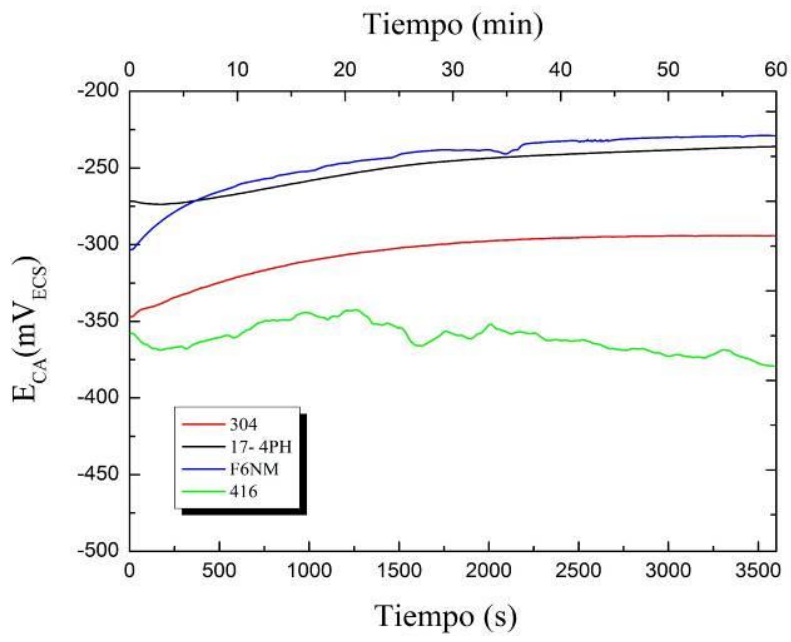

Figura 1: Variación del circuito abierto en el tiempo.

En la tabla 2 se presentan los valores de resistencia a la polarización ( $\mathrm{Rp}_{\mathrm{POL}}$ ) obtenidos por el Método de Polarización lineal y las pendientes de Tafel (ba y bc). Los valores obtenidos para estas variables se encuentran en el rango de valores encontrados en la bibliografía para estos materiales. Se observó que la aleación 416 presentó el valor más bajo de $\mathrm{Rp}_{\mathrm{POL}}$, seguida por la aleación F6NM y la aleación 17-4PH, mientras que la aleación 304 presentó el valor más alto de RpPOL. 
Tabla 2: Valores de Rp obtenidos por Método de Polarización lineal.

\begin{tabular}{c|c|c|c|c|c}
\hline ALEACION & PREN & $\begin{array}{c}\text { Ecorr } \\
\left(\mathbf{m V}_{\text {ECS }}\right)\end{array}$ & $\begin{array}{c}\mathbf{R p P O L} \\
\left(\mathbf{\Omega . c m}^{2}\right)\end{array}$ & $\begin{array}{c}\text { ba } \\
(\mathbf{m V})\end{array}$ & $\begin{array}{c}\text { bc } \\
(\mathbf{m V})\end{array}$ \\
\hline 416 & 13 & $-379,5$ & $5,58 \mathrm{E}+04$ & 115 & 130 \\
\hline F6NM & 15 & -240 & $1,83 \mathrm{E}+05$ & 160 & 185 \\
\hline $17-4 \mathrm{PH}$ & 17 & $-252,5$ & $2,05 \mathrm{E}+05$ & 245 & 75 \\
\hline 304 & 18 & $-301,5$ & $3,23 \mathrm{E}+05$ & 165 & 95 \\
\hline
\end{tabular}

En la figura 2 se presentan los diagramas de Bode y Nyquist obtenidos en los ensayos de EIE. Para todas las aleaciones se evidencia una sola contribución capacitiva, por lo cual el ajuste de los datos se llevó a cabo utilizando el circuito equivalente que se presenta en la figura 3 [24, 25]. El parámetro $\mathrm{R}_{\Omega}$ indica la resistencia de la solución, $\mathrm{Rp}_{\mathrm{EIE}}$ representa la resistencia a la transferencia de carga y CPE es un elemento de fase constante. De manera formal, la impedancia de un CPE está dada por la Ecuación 3.

$$
Z(C P E)=Z_{0} \cdot(i w)^{-n}
$$

Cuando $n=0$, el CPE representa un resistencia pura; si $n=1$ el CPE representa una capacitancia pura; y si $n=0,5$ el CPE denota una impedancia Warburg [24, 25]. En la Tabla 3 se presentan los parámetros del ajuste de impedancia.

Los altos valores de $n$ obtenidos, muestras que el elemento de fase constante dentro de nuestro circuito actúa como un capacitor. Al igual que en los valores obtenidos a partir del método de Tafel, la aleación 416 fue la que presentó el menor valor de $\mathrm{Rp}_{\mathrm{EIE}}$.

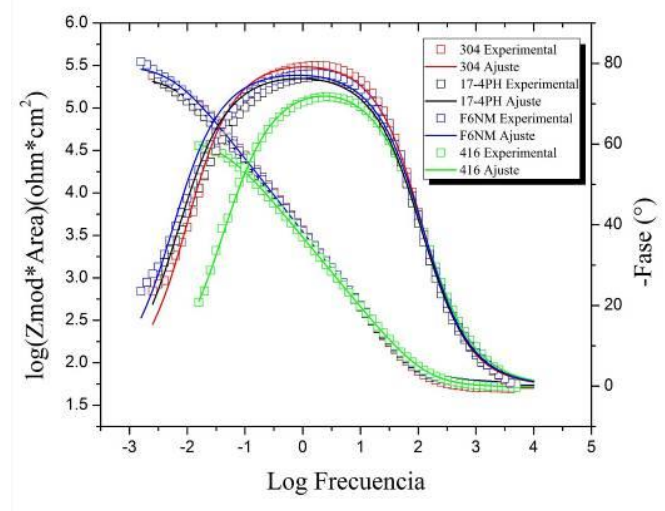

(a)

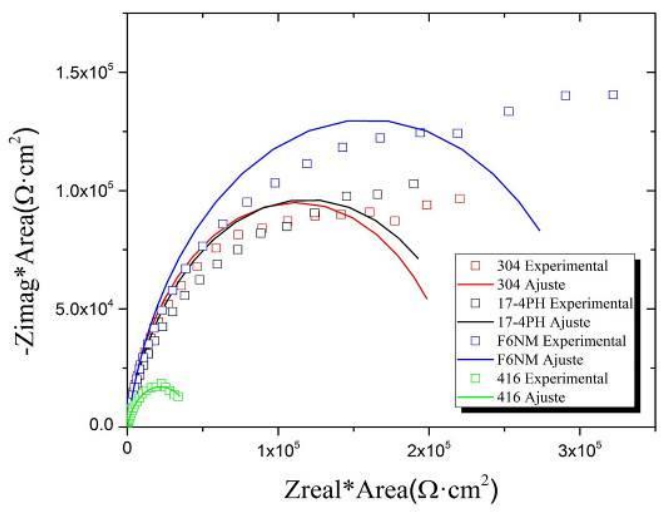

(b)

Figura 2: Diagrama de Bode (a) y Nyquist (b) obtenidos de los ensayos de EIE realizados a las aleaciones 304, 174HP, F6NM y 416 en .una solución de 10.000 ppm NaCl a temperatura ambiente. Símbolos: experimental. Líneas: ajuste

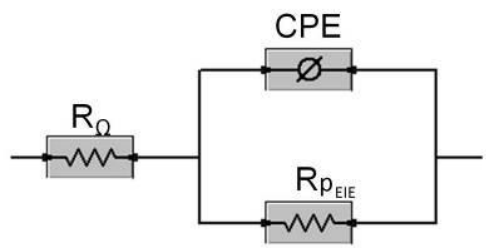

Figura 3: Circuito equivalente para el ajuste de los datos experimentales. 
Tabla 3: Parámetros de ajuste de impedancia obtenidos por EIE.

\begin{tabular}{c|c|c|c|c|c}
\hline ALEACION & PREN & $\begin{array}{c}\mathbf{R}_{\mathbf{\Omega}} \\
\left(\boldsymbol{\Omega} \cdot \mathbf{c m}^{2}\right)\end{array}$ & $\begin{array}{c}\mathbf{R} \mathbf{p}_{\mathrm{EIE}} \\
\left(\mathbf{\Omega} \cdot \mathbf{c m}^{2}\right)\end{array}$ & $\begin{array}{c}\mathbf{C P E} \\
\left(\mathbf{\Omega}^{-1} \cdot \mathbf{s}^{-\mathbf{n}} \cdot \mathbf{c m}^{-2}\right)\end{array}$ & $\boldsymbol{\eta}$ \\
\hline 416 & 13 & $37,87(2.93 \%)$ & $4,40 \mathrm{E}+04(7.05 \%)$ & $6,34 \mathrm{E}-05(3.85 \%)$ & $0,85(1.18 \%)$ \\
\hline F6NM & 15 & $37,91(2.97 \%)$ & $2,46 \mathrm{E}+05(7.10 \%)$ & $5,45 \mathrm{E}-05(2.72 \%)$ & $0,88(0.85 \%)$ \\
\hline $17-4 \mathrm{PH}$ & 17 & $38,32(2.96 \%)$ & $2,60 \mathrm{E}+05(7.27 \%)$ & $5,79 \mathrm{E}-05(2.84 \%)$ & $0,89(0.90 \%)$ \\
\hline 304 & 18 & $36,64(2.90 \%)$ & $2,86 \mathrm{E}+05(6.49 \%)$ & $5,07 \mathrm{E}-05(2.88 \%)$ & $0,90(0.32 \%)$ \\
\hline
\end{tabular}

(*) Los datos presentados entre paréntesis corresponden al error estimado en el ajuste

En la figura 4 se presentan las curvas de polarización cíclicas de las aleaciones 416, F6NM, 17-4PH y 304 obtenidas en una solución de $10.000 \mathrm{ppm} \mathrm{NaCl}$ a temperatura ambiente. Se observó que las aleaciones 304, F6NM y 17-4PH presentaron un potencial de corrosión (Ecorr) y una zona de pasividad similares entre sí, en tanto que la aleación 416 mostró una zona de pasividad a valores de densidad de corriente más altos y un potencial de corrosión $50 \mathrm{mV}$ menor que las demás. A partir de estas curvas se obtuvieron los valores del potencial de repasivasión $\left(\mathrm{E}_{\mathrm{R}}\right)$ y de picado $(\mathrm{Ep})$. Se definió el potencial de repasivasión $\left(\mathrm{E}_{\mathrm{R}}\right)$ como el potencial al cual la curva potenciodinámica de barrido inverso corta a la de barrido directo. En la tabla 4 se presenta los valores medios obtenidos para cada caso.

Luego del ensayo, se observaron las probetas en el microscopio óptico. Las micrografías obtenidas para los distintos materiales se presentan en la figura 5. Se advirtió que todas las aleaciones presentaron corrosión por picaduras, siendo las más profundas, las encontradas en las aleaciones F6NM y 416.

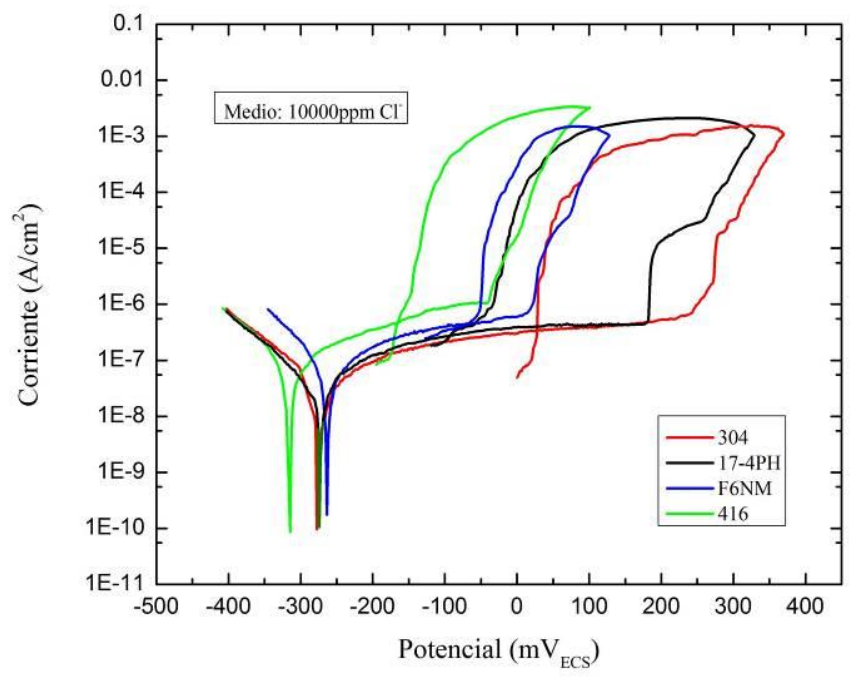

Figura 4: Curvas de polarización potenciodinámica de las aleaciones estudiadas.

Tabla 4: Curvas de polarización potenciodinámica de las aleaciones estudiadas.

\begin{tabular}{l|l|l|l|l}
\hline ALEACION & MICROESTRUCTURA & PREN & $\mathbf{E}_{\mathrm{R}}(\mathbf{m V})$ & E $_{\mathrm{P}}(\mathbf{m V})$ \\
\hline 416 & Martensítico & 13 & $-165,69$ & $-25,95$ \\
\hline F6NM & Martensítico & 15 & $-80,985$ & 7,8 \\
\hline $17-4 \mathrm{PH}$ & Martensítico PH & 17 & $-50,25$ & 167,21 \\
\hline 304 & Austenítico & 18 & 14,315 & 218,65 \\
\hline
\end{tabular}



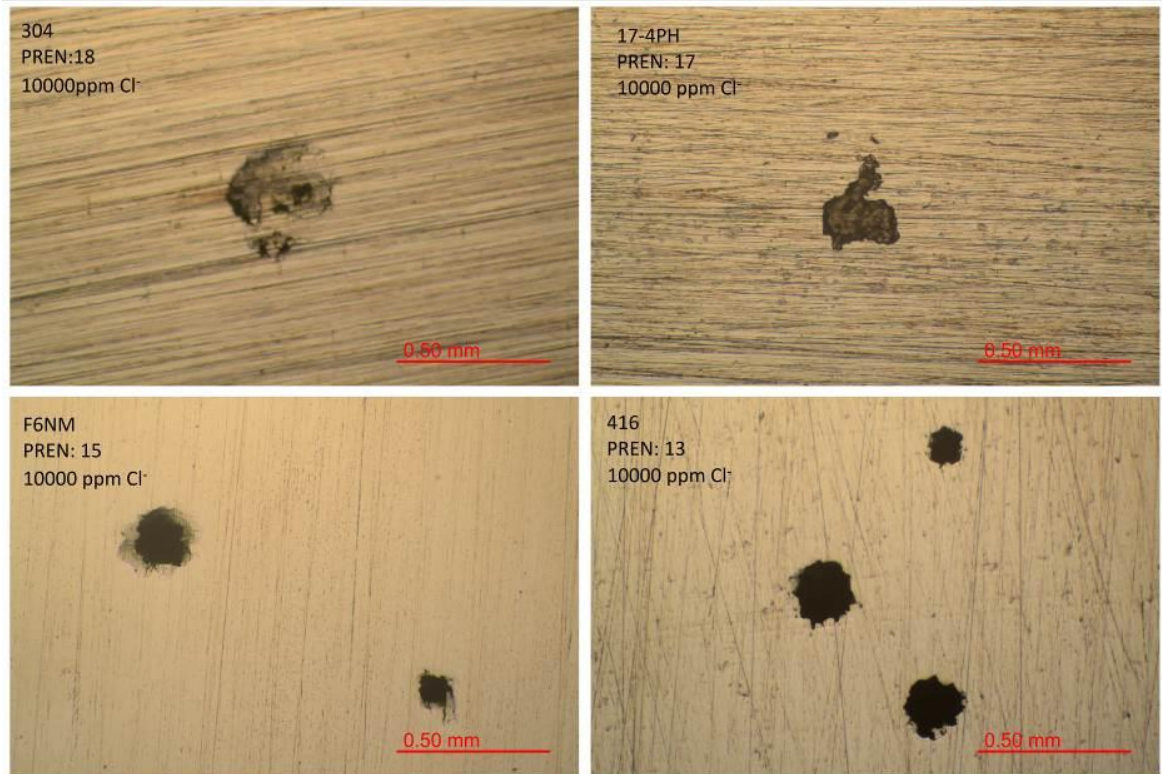

Figura 5: Micrografías de las probetas luego del ensayo de las curvas de polarización.

\section{DISCUSIÓN}

A partir del registro del potencial de circuito abierto en el tiempo se pudo evidenciar que, de todas las aleaciones estudiadas en este trabajo, la aleación 416 fue la que presentó el comportamiento más desfavorable en comparación con los demás materiales. El alto valor del $\mathrm{E}_{\mathrm{CA}}$ que presentó permitió anticipar que las aleaciones 304, F6NM y 17-4PH exhibirían un mejor comportamiento bajo estas condiciones. Esto se evidenció al evaluar los valores de resistencia a la transferencia de carga (Rp), obtenidas tanto por el método de Tafel, como de las medidas de EIE.

La resistencia a la transferencia de carga es usualmente usada como una medida de la resistencia del material frente a la corrosión. Aquellos materiales con mayores valores de Rp representan a aquellos con mejor comportamiento frente a la corrosión. Del análisis realizado a los valores de resistencia a la transferencia de carga, se observó que la aleación 416 es más susceptible a sufrir corrosión que el resto de las aleaciones. Al mismo tiempo, se encontró una dependencia de la resistencia a la transferencia de carga en función del PREN, independientemente de sus microestructuras. En la figura 6 se presenta la variación de los valores de $\mathrm{Rp}$ obtenidos por el método de Tafel ( $\mathrm{R} \mathrm{p}_{\mathrm{POL}}$ ) y por medio de las medidas de EIE ( $\mathrm{Rp}_{\mathrm{EIE}}$ ) para las aleaciones 416, F6NM, 17-4PH y 304.

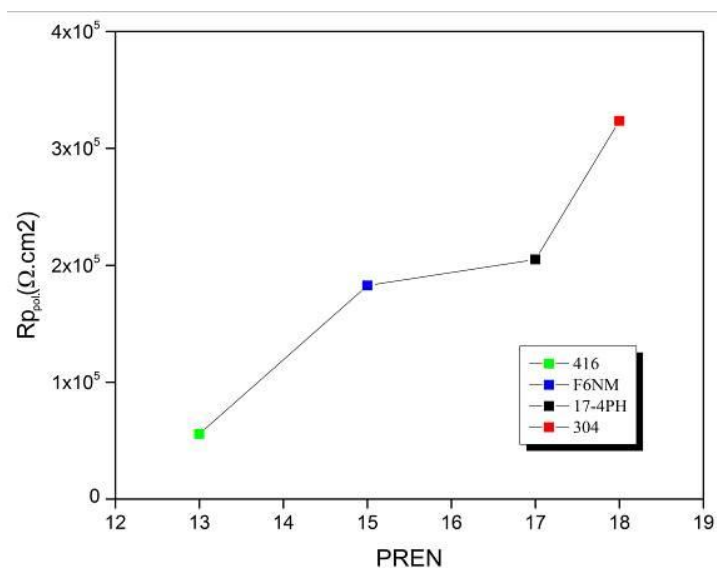

(a)

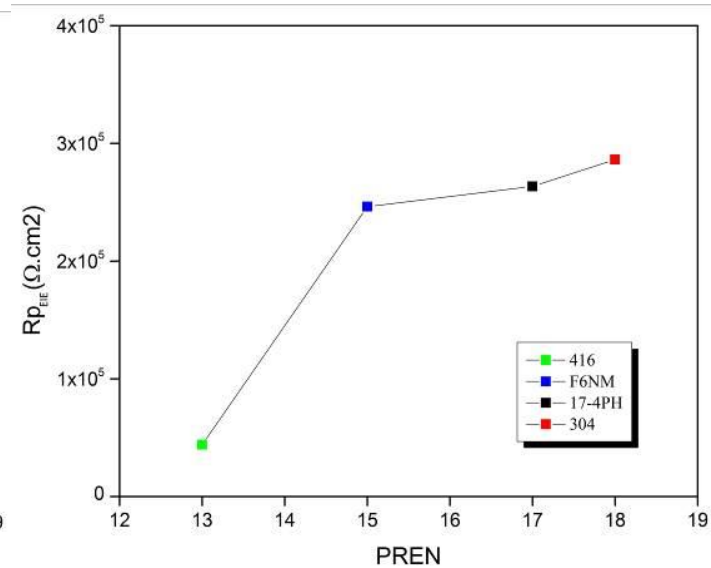

(b)

Figura 6: Variación de los valores de Rp obtenidos por el método de (a) Tafel ( $\mathrm{Rp}_{\mathrm{POL}}$ ) y (b) por medio de las medidas de EIE ( $\left(\mathrm{Rp}_{\mathrm{EIE}}\right)$ para las aleaciones 416, F6NM, 17-4PH y 304.

Si bien los valores obtenidos en ambos métodos no son iguales, esta tendencia se presenta en ambos casos. Además, se observó que entre las aleaciones F6NM y 17-4PH (martensíticos) la resistencia a la polari- 
zación es bastante similar, a pesar de que la aleación 17-4PH tiene un PREN mayor. Esto puede deberse a que el tratamiento térmico de endurecimiento por precipitación de esta aleación produjo una disminución en su resistencia a la corrosión. Para evaluar la resistencia a la corrosión localizada, se analizó los valores de potenciales de repasivasión $\left(E_{R}\right)$ y potenciales de picado $\left(E_{P}\right)$ en función del PREN (tabla 4). Se observó que estos potenciales fueron mayores para la aleación 304, seguido por las aleaciones 17-4PH, F6NM y 416. Nuevamente el comportamiento de estos materiales frente a la corrosión muestra una tendencia en la cual, a medida que aumenta el valor del PREN, aumenta la resistencia a la corrosión localizada. No se encuentra ninguna influencia de la microestructura de las aleaciones en su resistencia a la corrosión.

\section{CONCLUSIONES}

El Método de polarización lineal y las medidas de espectroscopía de impedancia electroquímica (EIE) indicaron que la resistencia a la polarización es mayor en las aleaciones con valores de PREN más altos, independientemente de su microestructura. Aunque hay cierta discrepancia entre los valores obtenidos por ambos métodos, esta tendencia se mantiene en ambos casos.

El análisis de las curvas de polarización potenciodinámicas cíclicas permite concluir que hay una dependencia de los potenciales de picado y de la repasivación con el PREN.

No se encontró evidencia de que la microestructura que presentan las aleaciones estudiadas tenga algún tipo de influencia sobre la resistencia a la corrosión.

\section{BIBLIOGRAFÍA}

[1] NALLI K, Corrosion and its mitigation in the oil and gas industry. An overview. PM-Pipeliner Report. 2010.

[2] MANNAN S, PATEL S, A new high strength corrosion resistant alloy for oil and gas applications. Paper presented at NACE Corrosion, New Orleans, 2008.

[3] CRAIG BD, Selection guidelines for corrosion resistant alloys in the oil and gas industry. NiDI Tech Series 10073, pp. 1-8, 1995.

[4] SINGH R., Applied Welding Engineering: Processes, Codes and Standards Applied Welding Engineering: Processes, Codes and Standards, First edit, Elsevier Inc, doi:10.1016/B978-0-12-391916-8.X000, pp 1$5,2012$.

[5] MOORE P., The Atlas Steels Technical Handbook of Stainless Steels, Atlas Steels Technical Department, 2013.

[6] ECHEVERRIA M.I, SOLÍS, J.M.H. IBÁÑEz, Manual Aceros Inoxidables, @INDURA S.A., Cerrillos, Chile, 2010.

[7] Handbook of Stainless Steel, Outokumpu Oyj, 2013

[8] GALVELE J. R. y DUFFÓ G. S., Degradación de Materiales. Corrosión. Primera Edición, Jorge Baudino Ediciones, Instituto Sabato, Buenos Aires, 2006.

[9] FONTANA M., Corrosion Engineering, Third edition, McGraw-Hill, 1986.

[10] Uhlig's Corrosion Handbook, Second Edition, Edited by R. Winston Revie, John Wiley \& Sons, Inc., 2000.

[11] CORROSION, v. 1, Metal/Environment Reactions, edited by, L. L. Shreir, L. A. Jarman and G. T. Burstein, Third edition, 2000.

[12] SZKLARSKA-SMIALOWSKA Z., Pitting and Crevice Corrosion, NACE Intl, 2005

[13] FRANKEL G. S., "Pitting Corrosion of Metals. A review of the critical factors", Journal of The Electrochemical Society, v. 145, n. 6, pp. 2186-2198, 1998.

[14] SEDRIKS A. J., Corrosion of stainless steels, Second edition, John Wiley \& Sons, Inc., 1996

[15] JSSELING F. P. I., Electrochemical methods in crevice corrosion testing, British Corrosion Journal, Vol. $15, \mathrm{~N}^{\circ} 2$, pp. 51-69, 1980.

[16] REBAK R. B., in Materials science and technology. A comprehensive treatment. Corrosion and environmental degradation, v.II, pp. 69-111, Wiley, VCH, 2000.

[17] AGARWAL D. C., Nickel and nickel alloys in Uhlig's Corrosion Handbook, Second Edition, edited by 
R. Winston Revie, John Wiley \& Sons, Inc. 2003.

[18] COMBRADE P., Crevice corrosion of metallic materials in Corrosion mechanisms in theory and practice, Marcel Dekker, pp. 349-397, 2000.

[19] REBAK R. B. and CROOK P., Improved Pitting and Crevice Corrosion Resistance of Nickel and Cobalt Based Alloys, ECPV 98-17, The Electrochemical Society, Pennington York, NJ, pp. 289-302, 1999.

[20] ASTM G5-94(2004), "Standard Reference Test Method for Making Potentiostatic and Potentiodynamic Anodic Polarization Measurements", In: Annual Book of ASTM Standards, vol. 03.02 (West Conshohocken, PA: ASTM Intl., 2004), pp. 53-64

[21] ASTM-G59-97. Standard Test Method for Conducting Potentiodynamic Polarization Resistance Measurements. Annual book of ASTM standards.

[22] BOUKAMP B. A., Equivalent Circuit, University of Twente, 1989.

[23] LORENZ W., "Determination of corrosion rates by electrochemical DC and AC methods", Corros Sci n.9, pp. 647-672, 1981.

[24] ZADOROZNE, N.S., VIER, J.D., REBAK, R.B, et al., "Corrosion Behavior of Super-Ferritic

Stainless Steels in NaCl Media”, The Minerals, Metals \& Materials Society, pp 339-348, 2017.

[25] ZADOROZNE, N.S., REBAK, R.B, "Localized Corrosion Behavior of Ferritic and Austenitic Passive Materials Stainless Steels in NaCl Media", In: Corrosion, NACE International Conference and Expo, Louisiana EEUU, 2017. 